\section{Bayram DELES'}

Department of Child Development, Institute of Health Sciences

Ankara University Ankara, Turkey

Prof. Dr. Nilgun SARP ${ }^{2}$

Department of Child Development, Faculty of Health Sciences

Bilgi University Istanbul, Turkey
Original scientific paper

UDC: 371.78

DOI: $10.5937 /$ IstrPed2101124D

\title{
INVESTIGATION OF THE CORRELATION BETWEEN CHILDHOOD TRAUMA, PSYCHOLOGICAL WELL-BEING, AND ALTRUISTIC FEAR OF CRIME OF MOTHERS WITH CHILDREN AGED THREE TO SIX YEARS
}

\begin{abstract}
Childhood is the period during which physical and mental development is extremely rapid from the first years of life. Furthermore, many basic factors affecting the future lives of individuals start to be shaped. The child's future life, psychological well-being, and quality of life are negatively affected by the exposure to unwanted situations by the family or the environment during this period. As a matter of fact, behavior patterns exhibited by individuals during adulthood are thought to be correlated with childhood traumas. Thus, this study aimed to investigate the relationship between the altruistic fear of crime, psychological well-being, and childhood traumas among mothers with children aged three to six years. A crosssectional correlation research design was used for this study. The sample of the study consisted of 214 volunteer mothers, whose children were attending a private preschool education institution in the city center of Gumushane province of Turkey during the 2019/2020 academic year. The research data were collected using "Personal Information Form", "Childhood Trauma Questionnaire", "Altruistic Fear of Crime Scale" and "Psychological Well-being Scale". Data were analyzed using t-test, ANOVA, and correlation analysis. The results of the research showed that the age of the mothers, birth order, presence of any family member receiving psychological support, and marital satisfaction caused significant differences. Furthermore, scores obtained from "Personal Information Form", "Childhood Trauma Questionnaire", "Altruistic Fear of Crime" and "Psychological Well-being Scale" were found to be significantly correlated.
\end{abstract}

Keywords: Childhood Traumas, Psychological Well-being, Altruistic Fear of Crime.

\section{Introduction}

Starting from the first days of life, individuals tend to establish social relationships with the people around them. They are dependent on the person, who is responsible for their care, to meet their needs, particularly during infancy and early childhood (Bruhn, 2010; Isgor, 2017). The relationships that the individual has established during childhood must be reliable (Roothman, Kirsten \& Wissing, 2003) for the individual to develop a sense of attachment. Attachment can influence the relationships that continue from an early age until the end of

1 delesbayram@gmail.com

nilgun.sarp@bilgi.edu.tr 
life (Medrano et al., 2002). Relationships established during childhood have cognitive, spiritual, physical, and emotional effects (Ryff \& Singer, 2008).

Adverse events or traumas experienced by individuals during childhood negatively affect both the childhood and later life of the individual (Medrano et al., 2002). Traumas are undesirable situations that can cause physical and mental harm to the individual, disrupt the integrity of the individual, and significantly differentiate attitudes and behaviors (Gopalakrishnan \& Sundram, 2014). Traumas experienced particularly in early years and critical periods of development can lead to greater and permanent effects on individuals. Sexual, emotional, or physical abuse and neglect are among the types of trauma that children are exposed to during childhood (Vozmediano et al., 2017). Many studies have reported that neglect and abusive behaviors have the same negative effects on individuals (Ozen, 2010; Yildiz, 2020). Negative situations and traumas experienced by individuals can cause altruistic fear of crime in individuals and damage their psychological well-being (Oymak, 2017; Talu \& Avci, 2019).

Psychological well-being refers to the individual's experience, functionality, and life satisfaction (Portnova \& Sivolap, 2017). The absence of negative emotions is one of the indicators of psychological well-being (Martha et al., 2002; Ozmete, 2016). People with high psychological well-being can establish healthy communication and relationship with others. The behavioral, cognitive, and social well-being of the individual is expressed with the concept of psychological well-being (Roothman, Kirsten \& Wissing, 2003). Psychological well-being has positive effects on life satisfaction (Tuzgol Dost, 2004). Individuals with high psychological well-being can be happy, hopeful, positive, optimistic, successful, and self-confident (Oymak, 2017). The presence of psychological well-being in individuals ensures the development of positive characteristics in the individual, including self-acceptance, the ability to make his/her own decisions and to organize relations with people around him/her, having a purpose in life, and self-development (Christopher, 1999; Ryff\& Singer, 2008).

Perceiving childhood traumas according to individual characteristics and being affected by them may also vary (Bruhn, 2010; Christopher, 1999; Isgor, 2017). Therefore, abusive behaviors or neglect that are considered to be risk factors cannot be generalized (Medrano et al., 2002). The literature review has shown that childhood traumas negatively affect the individual in psychological terms. Therefore, this study aimed to reveal the correlation between childhood traumas, psychological well-being, and altruistic fear of crime of mothers with children aged three to six years. Also, it is aimed to reveal significant differencies according to research variables. Answers to the following subproblems in the research were sought:

1. Is there a significant difference between the age variable and the childhood traumas, psychological well-being, and altruistic fear of crime?

2. Is there a significant difference between the presence of any family member receiving psychological support and childhood traumas, psychological well-being, and altruistic fear of crime?

3. Is there a significant difference between the birth order and the childhood traumas, psychological well-being, and altruistic fear of crime?

4. Is there a significant difference between marital satisfaction and childhood traumas, psychological well-being, and altruistic fear of crime?

5. Is there a significant correlation between childhood traumas, psychological wellbeing, and altruistic fear of crime? 


\section{Method}

\subsubsection{Model}

This study aimed to investigate the relationship between the altruistic fear of crime, psychological well-being, and childhood traumas among mothers with children aged three to six years. A cross-sectional correlation research design, which was one of the general survey models within the quantitative research methods, was used for this study. A general survey model is a research approach that aims to define the individuals or objects included in the study as they exist under their own conditions. In cross-sectional studies, any event is investigated within a specific time period (Karadag, 2010).

\subsubsection{Population and Sample}

The study population consisted of mothers, whose children were attending a private preschool education institution in the city center of Gumushane province of Turkey during the 2019/2020 academic year. Sampling was made through the accessible sampling method. A total of 214 mothers voluntarily participated in the study. Scales were sent and applied through the electronic environment.

\subsubsection{Data Collection Tools}

Data were collected using "Personal Information Form", "Childhood Trauma Questionnaire", "Psychological Well-being Scale", and "Altruistic Fear of Crime Scale".

Personal Information Form. This form consisted of questions about the mothers' age, educational status, birth order, presence of any family member receiving psychological support, and general marital satisfaction. The personal information form is prepared the researcher.

Childhood Trauma Questionnaire. It was developed by Bernstein et al. in 1994 and adapted into Turkish by Sar, Ozturk, and Ikikardes (2012). This five-point Likert-type scale consists of 28 items and five sub-dimensions (Physical abuse, emotional abuse, sexual abuse, physical neglect, and emotional neglect). Cronbach's alpha reliability coefficient of the overall scale was found to be .88 , and it was evaluated according to sub-dimensions.

Psychological Well-Being Scale. It was developed by Diener, Scollon and Lucas (2009) and adapted into Turkish by Telef (2013). The scale consists of eight items, each being scored from 1 to 5 . It is of a single-factor structure. The reliability coefficient of the scale (Cronbach's alpha value) was found to be 87 .

Altruistic Fear of Crime Scale. This five-point Likert type scale, which was developed by Talu and Avci (2019), consists of 20 items and is of single-factor structure. The explained total variance of the scale was $51.43 \%$ and the Cronbach's Alpha value was .92.

\subsubsection{Statistical Analysis}

Research data were analyzed using quantitative data analysis methods. The data were analyzed using SPSS version 24 software. Parametric statistical methods were used since the data were observed to be normally distributed (Buyukozturk, 2012). In this regard, t-tests, ANOVA, and correlation analyses were used. 


\section{Results}

Table 1. Distribution of Mothers by Age and Educational Status Variables

\begin{tabular}{llrr}
\hline & & Frequency & Percentage \\
\hline \multirow{4}{*}{ Age } & $19-24$ & 7 & 3.3 \\
& $25-30$ & 59 & 27.6 \\
& $31-35$ & 89 & 41.6 \\
& $36-40$ & 43 & 20.1 \\
\hline \multirow{4}{*}{ Educational Level } & & 16 & 7.5 \\
\hline & Primary school & 15 & 7.0 \\
& graduate & & \\
& Secondary school & & 13.6 \\
& graduate & 29 & 17.3 \\
& High school graduate & 37 & 62.1 \\
& University graduate & 133 & 100.0 \\
\hline
\end{tabular}

Assessment of the age distribution showed that 3.3\%, 27.6\%, 41.6\%, and $20.1 \%$ of mothers participating in the research were within the age group of 19-24 years, 25-30 years, 31-35 years, and 36-40 years, respectively, whereas $7.5 \%$ were found to be $\geq 41$ years of age. Of the mothers, $7 \%$ were found to be primary school graduates, $13.6 \%$ secondary school, $17.3 \%$ high school, and $62.1 \%$ university graduates.

Table 2. Comparison of Mothers' Childhood Traumas, Altruistic Fear of Crime, and Psychological Well-Being Scores by Age Variable

\begin{tabular}{|c|c|c|c|c|c|c|c|}
\hline & Age & $\mathrm{N}$ & $x$ & SD & sd & $\mathrm{F} / \mathrm{t}$ & $\mathrm{P}$ \\
\hline \multirow{6}{*}{ CTQ-Emotional Abuse } & $19-24$ years & 7 & 5.29 & 0.488 & \multirow{6}{*}{4} & \multirow{6}{*}{2.517} & \multirow{6}{*}{.043} \\
\hline & $25-30$ years & 59 & 6.42 & 2.581 & & & \\
\hline & $31-35$ years & 89 & 7.42 & 2.903 & & & \\
\hline & $36-40$ years & 43 & 6.44 & 1.843 & & & \\
\hline & $\geq 41$ years & 16 & 6.38 & 2.705 & & & \\
\hline & Total & 214 & 6.80 & 2.608 & & & \\
\hline \multirow{6}{*}{ CTQ-Physical Abuse } & $19-24$ years & 7 & 5.00 & 0.000 & \multirow{6}{*}{4} & \multirow{6}{*}{3.214} & \multirow{6}{*}{.014} \\
\hline & $25-30$ years & 59 & 5.42 & 0.675 & & & \\
\hline & $31-35$ years & 89 & 5.84 & 1.278 & & & \\
\hline & $36-40$ years & 43 & 5.51 & 1.055 & & & \\
\hline & $\geq 41$ years & 16 & 5.13 & 0.342 & & & \\
\hline & Total & 214 & 5.58 & 1.044 & & & \\
\hline \multirow{6}{*}{ CTQ-Physical Neglect } & $19-24$ years & 7 & 7.43 & 0.787 & \multirow{6}{*}{4} & \multirow{6}{*}{2.414} & \multirow{6}{*}{.050} \\
\hline & $25-30$ years & 59 & 8.97 & 3.459 & & & \\
\hline & 31-35 years & 89 & 8.01 & 2.794 & & & \\
\hline & $36-40$ years & 43 & 7.60 & 2.709 & & & \\
\hline & $\geq 41$ years & 16 & 6.94 & 1.731 & & & \\
\hline & Total & 214 & 8.09 & 2.922 & & & \\
\hline \multirow{6}{*}{ CTQ-Sexual Abuse } & $19-24$ years & 7 & 5.00 & 0.000 & \multirow{6}{*}{4} & \multirow{6}{*}{3.258} & \multirow{6}{*}{.013} \\
\hline & $25-30$ years & 59 & 5.61 & 1.992 & & & \\
\hline & $31-35$ years & 89 & 6.03 & 2.086 & & & \\
\hline & $36-40$ years & 43 & 5.23 & 0.718 & & & \\
\hline & $\geq 41$ years & 16 & 7.13 & 3.845 & & & \\
\hline & Total & 214 & 5.80 & 2.067 & & & \\
\hline \multirow{4}{*}{ Psychological Well-Being } & $19-24$ years & 7 & 50.71 & .487 & 4 & \multirow{4}{*}{2.222} & \multirow{4}{*}{.068} \\
\hline & $25-30$ years & 59 & 45.13 & 8.402 & & & \\
\hline & $31-35$ years & 89 & 47.91 & 8.074 & & & \\
\hline & $36-40$ years & 43 & 47.23 & 5.480 & & & \\
\hline
\end{tabular}




\begin{tabular}{|c|c|c|c|c|c|c|c|}
\hline & $\geq 41$ years & 16 & 43.56 & 11.488 & & & \\
\hline & Total & 214 & 46.77 & 7.999 & & & \\
\hline \multirow{6}{*}{ Altruistic Fear of Crime } & $19-24$ years & 7 & 27.42 & 2.699 & \multirow{6}{*}{4} & \multirow{6}{*}{1.779} & \multirow{6}{*}{.134} \\
\hline & $25-30$ years & 59 & 28.11 & 6.406 & & & \\
\hline & $31-35$ years & 89 & 30.46 & 8.090 & & & \\
\hline & $36-40$ years & 43 & 31.51 & 7.225 & & & \\
\hline & $\geq 41$ years & 16 & 30.18 & 5.867 & & & \\
\hline & Total & 214 & 29.90 & 7.272 & & & \\
\hline
\end{tabular}

The comparison of scores obtained from the Childhood Trauma Questionnaire according to the age variable was performed using ANOVA. The analysis showed that there was a significant difference between mothers in different age groups $(p \leq 05)$. As a result of the Tukey test, scores obtained by the mothers aged 31-35 years from emotional abuse, physical abuse, and sexual abuse subscales were found to be significantly higher than those of mothers in the age groups of 25-30 and 36-40 years. Physical neglect scores of mothers aged 25-30 years were found to be significantly higher than those of mothers in the age groups of 31-35 and 36-40 years. There was no significant difference between age groups in terms of the scores obtained from altruistic fear of crime and psychological well-being scales ( $p>05)$.

Table 3. Comparison of Mothers' Childhood Traumas, Altruistic Fear of Crime And Psychological Well-Being Scores by The 'Presence of Any Family Member Receiving Psychological Support' Variable

\begin{tabular}{|c|c|c|c|c|c|c|c|}
\hline & $\begin{array}{l}\text { Did any of your family members } \\
\text { receive psychological support? }\end{array}$ & $\mathrm{N}$ & $x$ & SD & sd & $\mathrm{F}$ & $\mathrm{P}$ \\
\hline $\begin{array}{l}\text { CTQ-Emotional } \\
\text { Abuse }\end{array}$ & $\begin{array}{l}\text { My mother received } \\
\text { psychological support } \\
\text { My father received psychological } \\
\text { support } \\
\text { My brother/sister received } \\
\text { psychological support } \\
\text { None of my family members } \\
\text { received psychological support } \\
\text { Total }\end{array}$ & $\begin{array}{r}4 \\
184 \\
214\end{array}$ & $\begin{array}{l}7.13 \\
7.00 \\
6.50 \\
6.80\end{array}$ & $\begin{array}{l}3.308 \\
1.885 \\
1.414 \\
2.414 \\
2.608\end{array}$ & 3 & 9.030 & .000 \\
\hline $\begin{array}{l}\text { CTQ-Emotional } \\
\text { Neglect }\end{array}$ & $\begin{array}{l}\text { My mother received } \\
\text { psychological support } \\
\text { My father received psychological } \\
\text { support } \\
\text { My brother/sister received } \\
\text { psychological support } \\
\text { None of my family members } \\
\text { received psychological support } \\
\text { Total }\end{array}$ & $\begin{array}{r}4 \\
184 \\
214\end{array}$ & $\begin{array}{r}13.11 \\
16.25 \\
12.00 \\
9.60 \\
10.19\end{array}$ & $\begin{array}{l}2.928 \\
5.007 \\
4.000 \\
3.283 \\
3.669\end{array}$ & 3 & 15.695 & .000 \\
\hline CTQ-Sexual Abuse & $\begin{array}{l}\text { My mother received } \\
\text { psychological support } \\
\text { My father received psychological } \\
\text { support } \\
\text { My brother/sister received } \\
\text { psychological support } \\
\text { None of my family members } \\
\text { received psychological support } \\
\text { Total }\end{array}$ & $\begin{array}{l}184 \\
214\end{array}$ & $\begin{array}{l}7.28 \\
8.00 \\
6.50 \\
\\
5.55 \\
5.80\end{array}$ & $\begin{array}{l}3.322 \\
2.777 \\
1.000 \\
1.770 \\
2.067\end{array}$ & 3 & 7.836 & .000 \\
\hline $\begin{array}{l}\text { CTQ-Overprotection- } \\
\text { Overcontrol }\end{array}$ & $\begin{array}{l}\text { My mother received } \\
\text { psychological support } \\
\text { My father received psychological } \\
\text { support }\end{array}$ & 18 & $\begin{array}{r}5.17 \\
3.00\end{array}$ & $\begin{array}{l}1.654 \\
1.690\end{array}$ & 3 & 13.027 & .000 \\
\hline
\end{tabular}




\begin{tabular}{|c|c|c|c|c|c|c|c|}
\hline & $\begin{array}{l}\text { My brother/sister received } \\
\text { psychological support } \\
\text { None of my family members } \\
\text { received psychological support } \\
\text { Total }\end{array}$ & $\begin{array}{r}4 \\
184 \\
214\end{array}$ & $\begin{array}{l}2.25 \\
3.43 \\
3.54\end{array}$ & $\begin{array}{l}0.500 \\
1.167 \\
1.327 \\
\end{array}$ & & & \\
\hline $\begin{array}{l}\text { Psychological Well- } \\
\text { Being Scale }\end{array}$ & $\begin{array}{l}\text { My mother received } \\
\text { psychological support } \\
\text { My father received psychological } \\
\text { support } \\
\text { My brother/sister received } \\
\text { psychological support } \\
\text { None of my family members } \\
\text { received psychological support } \\
\text { Total }\end{array}$ & $\begin{array}{l}184 \\
214\end{array}$ & $\begin{array}{r}43.11 \\
40.50 \\
52.00 \\
\\
47.29 \\
46.78\end{array}$ & $\begin{array}{l}6.650 \\
6.481 \\
5.228 \\
8.026 \\
8.000\end{array}$ & 3 & 3.877 & .010 \\
\hline $\begin{array}{l}\text { Altruistic Fear of } \\
\text { Crime }\end{array}$ & $\begin{array}{l}\text { My mother received } \\
\text { psychological support } \\
\text { My father received psychological } \\
\text { support } \\
\text { My brother/sister received } \\
\text { psychological support } \\
\text { None of my family members } \\
\text { received psychological support } \\
\text { Total }\end{array}$ & $\begin{array}{l}184 \\
214\end{array}$ & $\begin{array}{l}30.35 \\
29.90\end{array}$ & $\begin{array}{l}3.399 \\
1.908\end{array}$ & 3 & 2.052 & .108 \\
\hline
\end{tabular}

The comparison of the scores obtained from the Childhood Trauma Questionnaire according to the 'presence of any family member receiving psychological support' variable was performed using ANOVA. A significant difference was observed as a result of the analysis $(\mathrm{p} \leq$ .05). Tukey test showed that women whose mothers had received psychological support were found to have higher scores on emotional abuse, emotional neglect, sexual abuse, and overprotection-overcontrol subscales compared to those who had no family members receiving psychological support. When the Psychological Well-Being Scale results were evaluated, those who had no family members receiving psychological support were found to have higher psychological well-being compared to participants whose mothers had received psychological support. There was no significant difference between the groups in terms of the scores obtained from the altruistic fear of the crime scale $(p>.05)$.

Table 4. Comparison of Mothers' Childhood Traumas, Altruistic Fear of Crime, and Psychological Well-Being Scores by the Birth Order Variable

\begin{tabular}{llrrrrrr}
\hline & $\begin{array}{l}\text { Ordinal Position } \\
\text { Among the Children } \\
\text { in the Family }\end{array}$ & $\mathrm{N}$ & $\mathrm{X}$ & SD & sd & F & P \\
& 1 & 82 & 7.18 & 2.846 & & & \\
CTQ-Emotional & 2 & 67 & 6.42 & 2.097 & & & \\
Abuse & 3 & 30 & 7.67 & 3.437 & 4 & 3.006 & .019 \\
& 4 & 11 & 6.45 & 1.809 & & & \\
& 5 and above & 24 & 5.63 & 1.439 & & & \\
& Total & 214 & 6.80 & 2.608 & & & \\
& 1 & 82 & 5.78 & 1.217 & & & \\
CTQ-Physical & 2 & 67 & 5.51 & 1.092 & & & \\
Abuse & 3 & 30 & 5.70 & 0.794 & 4 & 2.609 & .037 \\
& 4 & 11 & 5.27 & 0.467 & & & \\
& 5 and above & 24 & 5.08 & 0.282 & & & \\
\hline
\end{tabular}




\begin{tabular}{|c|c|c|c|c|c|c|c|}
\hline \multirow{6}{*}{$\begin{array}{l}\text { CTQ-Physical } \\
\text { Neglect }\end{array}$} & 1 & 82 & 7.80 & 3.109 & \multirow{6}{*}{4} & \multirow{6}{*}{3.211} & \multirow{6}{*}{.014} \\
\hline & 2 & 67 & 7.57 & 2.401 & & & \\
\hline & 3 & 30 & 9.47 & 3.159 & & & \\
\hline & 4 & 11 & 9.55 & 1.635 & & & \\
\hline & 5 and above & 24 & 8.17 & 3.185 & & & \\
\hline & Total & 214 & 8.09 & 2.922 & & & \\
\hline \multirow{6}{*}{$\begin{array}{l}\text { CTQ-Emotional } \\
\text { Neglect }\end{array}$} & 1 & 82 & 10.45 & 3.969 & \multirow{6}{*}{4} & \multirow{6}{*}{.318} & \multirow{6}{*}{.866} \\
\hline & 2 & 67 & 10.01 & 3.748 & & & \\
\hline & 3 & 30 & 10.40 & 3.001 & & & \\
\hline & 4 & 11 & 9.55 & 2.583 & & & \\
\hline & 5 and above & 24 & 9.79 & 3.718 & & & \\
\hline & Total & 214 & 10.19 & 3.669 & & & \\
\hline \multirow{6}{*}{$\begin{array}{l}\text { CTQ-Sexual } \\
\text { Abuse }\end{array}$} & 1 & 82 & 5.99 & 2.058 & \multirow{6}{*}{4} & \multirow{6}{*}{.704} & \multirow{6}{*}{.590} \\
\hline & 2 & 67 & 5.85 & 2.265 & & & \\
\hline & 3 & 30 & 5.40 & 1.037 & & & \\
\hline & 4 & 11 & 5.18 & 0.405 & & & \\
\hline & 5 and above & 24 & 5.83 & 2.823 & & & \\
\hline & Total & 214 & 5.80 & 2.067 & & & \\
\hline \multirow{6}{*}{$\begin{array}{l}\text { CTQ- } \\
\text { Overprotection- } \\
\text { Overcontrol }\end{array}$} & 1 & 82 & 3.76 & 1.560 & \multirow{6}{*}{4} & \multirow{6}{*}{1.856} & \multirow{6}{*}{.119} \\
\hline & 2 & 67 & 3.36 & 1.124 & & & \\
\hline & 3 & 30 & 3.70 & 1.055 & & & \\
\hline & 4 & 11 & 3.64 & 0.674 & & & \\
\hline & 5 and above & 24 & 3.04 & 1.398 & & & \\
\hline & Total & 214 & 3.54 & 1.327 & & & \\
\hline \multirow{6}{*}{$\begin{array}{l}\text { Psychological } \\
\text { Well-Being } \\
\text { Scale }\end{array}$} & 1 & 82 & 46.07 & 7.253 & \multirow{6}{*}{4} & \multirow{6}{*}{3.241} & \multirow{6}{*}{.013} \\
\hline & 2 & 67 & 48.54 & 4.640 & & & \\
\hline & 3 & 30 & 47.33 & 7.194 & & & \\
\hline & 4 & 11 & $49 \cdot 36$ & 11.111 & & & \\
\hline & 5 and above & 24 & 42.38 & 13.859 & & & \\
\hline & Total & 214 & 46.78 & 8.000 & & & \\
\hline \multirow{6}{*}{$\begin{array}{l}\text { Altruistic } \\
\text { of Crime }\end{array}$} & 1 & 82 & 30.35 & 7.000 & \multirow{6}{*}{4} & \multirow{6}{*}{.823} & \multirow{6}{*}{.512} \\
\hline & 2 & 67 & 30.13 & 7.156 & & & \\
\hline & 3 & 30 & 28.70 & 7.442 & & & \\
\hline & 4 & 11 & 26.91 & 10.261 & & & \\
\hline & 5 and above & 24 & 30.63 & 6.858 & & & \\
\hline & Total & 214 & 29.91 & 7.272 & & & \\
\hline
\end{tabular}

The comparison of the scores obtained from the childhood trauma questionnaire according to the birth order was performed using ANOVA. The analysis showed that there was a significant difference in emotional abuse, physical abuse, physical neglect, and psychological well-being scale scores $(p \leq .05)$. Tukey test revealed that first-borns were found to obtain higher scores on emotional abuse and physical abuse subscales compared to second-borns and the physical neglect levels were found to be higher among third- and fourth-borns compared to first- and second-borns. The psychological well-being of second-, third-, and fourth-borns were found to be higher than that of first-borns.

Table 5. Comparison of Mothers' Childhood Traumas, Altruistic Fear of Crime And Psychological Well-Being Scores by Marital Satisfaction Variable

\begin{tabular}{llcccccc}
\hline & Marital Satisfaction & N & X & SD & sd & F/t & P \\
\hline \multirow{2}{*}{ CTQ- } & I am very satisfied with my marriage & 115 & 5.99 & 1.694 & & & \\
Emotional & I am partially satisfied with my marriage & 89 & 7.66 & 3.030 & & & \\
Abuse & I am not satisfied with my marriage & 10 & 8.40 & 4.033 & & 13.757 & .000 \\
& Total & 214 & 6.80 & 2.608 & & & \\
\hline CTQ-Physical & I am very satisfied with my marriage & 115 & 5.37 & 0.821 & 2 & 5.035 & .007
\end{tabular}




\begin{tabular}{|c|c|c|c|c|c|c|c|}
\hline \multirow[t]{3}{*}{ Abuse } & I am partially satisfied with my marriage & 89 & 5.81 & 1.167 & & & \\
\hline & I am not satisfied with my marriage & 10 & 5.90 & 1.663 & & & \\
\hline & Total & 214 & 5.58 & 1.044 & & & \\
\hline \multirow{4}{*}{$\begin{array}{l}\text { CTQ-Physical } \\
\text { Neglect }\end{array}$} & I am very satisfied with my marriage & 115 & 7.23 & 2.090 & \multirow{4}{*}{2} & \multirow{4}{*}{13.265} & \multirow{4}{*}{.000} \\
\hline & I am partially satisfied with my marriage & 89 & 9.24 & 3.448 & & & \\
\hline & I am not satisfied with my marriage & 10 & 7.90 & 2.807 & & & \\
\hline & Total & 214 & 8.09 & 2.922 & & & \\
\hline \multirow{4}{*}{$\begin{array}{l}\text { CTQ- } \\
\text { Emotional } \\
\text { Neglect }\end{array}$} & I am very satisfied with my marriage & 115 & 8.77 & 2.791 & \multirow{4}{*}{2} & \multirow{4}{*}{22.919} & \multirow{4}{*}{.000} \\
\hline & I am partially satisfied with my marriage & 89 & 11.70 & 3.537 & & & \\
\hline & I am not satisfied with my marriage & 10 & 13.00 & 6.377 & & & \\
\hline & Total & 214 & 10.19 & 3.669 & & & \\
\hline \multirow{4}{*}{$\begin{array}{l}\text { CTQ-Sexual } \\
\text { Abuse }\end{array}$} & I am very satisfied with my marriage & 115 & $5 \cdot 32$ & 1.374 & \multirow{4}{*}{2} & \multirow{4}{*}{8.259} & \multirow{4}{*}{.000} \\
\hline & I am partially satisfied with my marriage & 89 & 6.46 & 2.667 & & & \\
\hline & I am not satisfied with my marriage & 10 & $5 \cdot 50$ & 0.527 & & & \\
\hline & Total & 214 & 5.80 & 2.067 & & & \\
\hline & I am very satisfied with my marriage & 115 & 48.75 & 7.953 & \multirow{4}{*}{2} & \multirow{4}{*}{8.071} & \multirow{4}{*}{.000} \\
\hline Well-Being & I am partially satisfied with my marriage & 89 & 44.44 & $7 \cdot 581$ & & & \\
\hline Scale & I am not satisfied with my marriage & 10 & 44.90 & 6.624 & & & \\
\hline & Total & 214 & 46.78 & 8.000 & & & \\
\hline \multirow{4}{*}{$\begin{array}{l}\text { Altruistic } \\
\text { Fear of Crime }\end{array}$} & I am very satisfied with my marriage & 115 & 30.66 & 7.476 & \multirow{4}{*}{2} & \multirow{4}{*}{$3 \cdot 326$} & \multirow{4}{*}{.038} \\
\hline & I am partially satisfied with my marriage & 89 & 28.55 & 6.945 & & & \\
\hline & I am not satisfied with my marriage & 10 & 33.30 & 5.813 & & & \\
\hline & Total & 214 & 29.91 & 7.272 & & & \\
\hline
\end{tabular}

The comparison of scores obtained from the childhood trauma questionnaire according to the marital satisfaction variable showed that there was a significant difference between the groups $(p \leq .05)$. Participants who were partially satisfied with their marriage were found to have higher levels of emotional abuse, physical abuse, physical neglect, emotional neglect, and sexual abuse compared to those who were very satisfied with their marriage. When the scores obtained from the psychological well-being scale and altruistic fear of crime scale were compared according to the marital satisfaction variable, those who were very satisfied with their marriage were found to have higher psychological well-being and lower fear of altruistic crime compared to those who were partially satisfied with their marriage.

Table 6. Correlation Between Childhood Traumas, Altruistic Fear of Crime And Psychological Well-Being Scores

\begin{tabular}{|c|c|c|c|c|c|c|c|c|c|}
\hline & & CTQ- & CTQ- & CTQ- & CTQ- & CTQ- & CTQ- & \multicolumn{2}{|c|}{ PsychologicalAltruistic } \\
\hline & & $\begin{array}{c}\text { Emotional } \\
\text { Abuse }\end{array}$ & $\begin{array}{l}\text { Physical } \\
\text { Abuse }\end{array}$ & $\begin{array}{l}\text { Physical } \\
\text { Neglect }\end{array}$ & $\begin{array}{c}\text { Emotiona } \\
\text { Neglect }\end{array}$ & $\begin{array}{l}\text { ISexualC } \\
\text { Abuse }\end{array}$ & $\begin{array}{l}\text { Dverprotection- } \\
\text { Overcontrol }\end{array}$ & $\begin{array}{c}\text { Well-Being } \\
\text { Scale }\end{array}$ & $\begin{array}{l}\text { Fear of } \\
\text { Crime }\end{array}$ \\
\hline \multirow{2}{*}{$\begin{array}{l}\text { CTQ-Emotional } \\
\text { Abuse }\end{array}$} & $r$ & 1 & & & & & & & \\
\hline & $\mathrm{P}$ & 214 & & & & & & & \\
\hline \multirow{3}{*}{$\begin{array}{l}\text { CTQ-Physical } \\
\text { Abuse }\end{array}$} & r & $.583^{*}$ & 1 & & & & & & \\
\hline & $\mathrm{p}$ & .000 & & & & & & & \\
\hline & $\mathrm{N}$ & 214 & 214 & & & & & & \\
\hline \multirow{3}{*}{$\begin{array}{l}\text { CTQ-Physical } \\
\text { Neglect }\end{array}$} & $r$ & $.467^{*}$ & $.524^{*}$ & 1 & & & & & \\
\hline & $\mathrm{p}$ & .000 & .000 & & & & & & \\
\hline & $\mathrm{N}$ & 214 & 214 & 214 & & & & & \\
\hline \multirow{3}{*}{$\begin{array}{l}\text { CTQ-Emotional } \\
\text { Neglect }\end{array}$} & $r$ & $.628^{*}$ & $.522^{*}$ & $.542^{*}$ & 1 & & & & \\
\hline & $\mathrm{p}$ & .000 & .000 & .000 & & & & & \\
\hline & $\mathrm{N}$ & 214 & 214 & 214 & 214 & & & & \\
\hline \multirow{3}{*}{$\begin{array}{l}\text { CTQ-Sexual } \\
\text { Abuse }\end{array}$} & $r$ & $.257^{*}$ & $.316^{*}$ & $.177^{*}$ & $.194^{*}$ & 1 & & & \\
\hline & $\mathrm{p}$ & .000 & .000 & .009 & .004 & & & & \\
\hline & $\mathrm{N}$ & 214 & 214 & 214 & 214 & 214 & & & \\
\hline
\end{tabular}




\begin{tabular}{|c|c|c|c|c|c|c|c|c|c|}
\hline CTQ- & $r$ & $.380^{*}$ & $.157^{*}$ & $.204^{*}$ & $.206^{*}$ & .033 & 1 & & \\
\hline Overprotection & & .000 & .022 & .003 & .002 & .626 & & & \\
\hline Overcontrol & $\mathrm{N}$ & 214 & 214 & 214 & 214 & 214 & 214 & & \\
\hline Psychological & $r$ & $-.187^{*}$ & -.079 & $-.242^{*}$ & $-.342^{*}$ & -.037 & -.016 & 1 & \\
\hline Well-Being & $\mathrm{p}$ & .006 & .248 & .000 & .000 & .593 & .821 & & \\
\hline Scale & $\mathrm{N}$ & 214 & 214 & 214 & 214 & 214 & 214 & 214 & \\
\hline \multirow{3}{*}{$\begin{array}{l}\text { Altruistic } \\
\text { of Crime }\end{array}$} & $r^{r}$ & $-.189^{*}$ & $-.135^{*}$ & $-.135^{*}$ & .112 & $-.154^{*}$ & -.058 & -.133 & 1 \\
\hline & $\mathrm{p}$ & .006 & .048 & .048 & .103 & .024 & .395 & .052 & \\
\hline & $\mathrm{N}$ & 214 & 214 & 214 & 214 & 214 & 214 & 214 & 214 \\
\hline
\end{tabular}

*. The correlation value was considered to be significant at the 0.05 level.

The correlation between childhood traumas, altruistic fear of crime, and psychological wellbeing scores were investigated using correlation analysis. As a result of this analysis, a significant correlation was observed between the scores $(p \leq .05)$. Considering the data presented in Table 6, a moderately positive significant correlation was present between the following Childhood Trauma Questionnaire sub-dimensions: physical abuse and emotional abuse; physical neglect and emotional \& physical abuse; emotional neglect and emotional \& physical abuse and physical neglect; sexual abuse and physical abuse; and overprotectionovercontrol and emotional abuse $(p \leq .05)$. Accordingly, as the emotional neglect levels of mothers increased, their levels of physical neglect, sexual abuse, physical abuse, emotional abuse, and overprotection-overcontrol increased. Furthermore, psychological well-being scale scores were found to have a weak negative significant correlation with emotional abuse and physical neglect scores and a moderately negative significant correlation with emotional neglect scores. According to this, increasing psychological well-being decreased emotional abuse, physical neglect, and emotional neglect levels. A weak negative and significant correlation was observed between the Altruistic Fear of Crime scale scores and emotional abuse, physical abuse, physical neglect, and sexual abuse scores $(p \leq .05)$. Considering that altruistic fear of crime scale scores was reverse-scored, emotional abuse, physical abuse, physical neglect, and sexual abuse levels were found to increase with the increasing altruistic fear of crime levels.

\section{Discussion}

The results of the present study have shown that the emotional abuse, physical abuse, and sexual abuse scores that the mothers in the age group of 31-35 years obtained from the Childhood Trauma Questionnaire were significantly higher than those of the mothers in the age group of 25-30 and 36-40 years. Furthermore, the physical neglect levels of mothers in the age group of 25-30 years have been observed to be higher compared to those in the age groups of 31-35 years and 36-40 years. On the other hand, no significant difference has been observed between age groups in terms of fear of crime and Psychological Well-Being Scale scores. In a study by Talu and Avci (2019) investigating mothers' altruistic fear of crime, altruistic fear of crime was reported to increase with the advancing age.

In the present study, it was observed that women whose mothers had received psychological support were found to have higher scores on emotional abuse, emotional neglect, sexual abuse, and overprotection-overcontrol subscales compared to those who had no family members receiving psychological support. When Psychological Well-Being Scale results were evaluated, those who had no family members receiving psychological support were found to have higher psychological well-being compared to participants whose mothers had received psychological support. 
It was further shown that first-borns were found to obtain higher scores on emotional abuse and physical abuse subscales compared to second-borns and the physical neglect levels were found to be higher among third- and fourth-borns compared to first- and second-borns. When analyzed in terms of birth order, there was no significant difference in psychological wellbeing and altruistic fear of crime scores. Similarly, Guler (2019) concluded that the birth order did have no effect on psychological well-being. Furthermore, Gopalakrishnan and Sundram (2014) and Bruhn (2010) also reported that psychological well-being did not differ according to birth order. Yildiz (2020) and Reinherz et al. (1993) reported that third-borns had higher levels of physical and emotional abuse than first- and second-borns in childhood traumas. Therefore, it can be said that the risk of being exposed to physical, emotional, or sexual trauma by other family members increases with the increasing birth order position.

In this study, participants who were partially satisfied with their marriage were found to have higher levels of emotional abuse, physical abuse, physical neglect, emotional neglect, and sexual abuse compared to those who were very satisfied with their marriage. Those who are very satisfied with their marriage have higher psychological well-being and lower altruistic fear of crime than those who are partially satisfied.

Emotional abuse, physical abuse, physical neglect, and sexual abuse levels were observed to increase as the levels of altruistic fear of crime increased. On the other hand, a negative correlation was observed between the increase in psychological well-being levels and childhood traumas. Similarly, Celebi and Polat (2019) investigated the effects of childhood traumas on marital satisfaction and attachment styles and concluded that individuals who experienced physical traumas and emotional or sexual abuse in childhood had lower marital satisfaction levels. Furthermore, Kim and McKenry (2002) reported a negative correlation between psychological well-being and childhood traumas. In a study by Martha et al. (2002), the effects of psychological disorders and childhood traumas on attachment were investigated. The authors concluded that there was a close relationship between the current state of the individual and all dimensions of childhood traumas. In a study by Vozmediano et al. (2017), in which the effects of family type and sex on altruistic fear of crime was investigated, sex was reported to be correlated with the crime type and women's altruistic fear levels were found to be higher than men. Beydogan Tangor and Curun (2016) investigated the predictors of psychological well-being and concluded that psychological wellbeing level negatively affected trait anxiety whereas increased self-esteem. In a study by Adiguzel, Ozdemir and Sahin (2019) investigating the effects of childhood traumas on suicide and aggression, the authors concluded that there was a significant correlation between all sub-dimensions of the Childhood Tauma Questionnaire and violence, bipolar disorders, and addictions. Portnova and Sivolap (2017) investigated the relationship between childhood trauma and substance abuse and reported that depression, anxiety, and other disorders were affected by childhood traumas. Campbell, Walker, and Egede (2016) concluded that exposure to trauma in childhood affected the risk of being a victim of abuse and violence later in life. In a study by Yildiz (2020), the physical, emotional, and sexual abuse sub-dimensions of childhood traumas were reported to be associated with stress and depression. Twaite and Rodriguez-Srednicki (2004) stated that physical and sexual abuse were associated with stress and depression and thus, psychological well-being was negatively affected by the presence of these two factors. 


\section{Conclusion and Recommendations}

The results of the present study are presented below.

The emotional abuse, physical abuse, and sexual abuse scores of mothers in the age group of 31-35 years are higher than those of the mothers in the age groups of 25-30 and 36-40 years whereas the physical neglect levels of mothers in the age group of 25-30 years are higher compared to the age groups of 31-35 and 36-40 years. Furthermore, women whose mothers are receiving psychological support have higher scores on emotional abuse, emotional neglect, sexual abuse, and overprotection-overcontrol subscales compared to those who have no family members receiving psychological support. First-borns have been found to obtain higher scores on emotional abuse and physical abuse subscales compared to secondborns and the physical neglect levels have been found to be higher among third- and fourthborns compared to first- and second-borns. Participants who are partially satisfied with their marriage have been found to have higher levels of emotional abuse, physical abuse, physical neglect, emotional neglect, and sexual abuse compared to those who are very satisfied with their marriage.

There was no significant difference in altruistic fear of crime and Psychological Well-Being Scale scores among age and birth order variables. The psychological well-being of women who have no family members receiving psychological support has been observed to be higher compared to those whose mothers received psychological support. Those who are very satisfied with their marriage have higher psychological well-being and lower altruistic fear of crime than those who are partially satisfied.

Emotional abuse, physical abuse, physical neglect, and sexual abuse levels increase as the levels of altruistic fear of crime increase. On the other hand, there has been a negative correlation between the increase in psychological well-being levels and childhood traumas. In line with these results, the following recommendations can be made:

$\checkmark \quad$ It is observed that childhood traumas negatively affect the later lives of individuals and thus, these negativities affect their psychological well-being. Therefore, parent training may be provided to prevent exposure to adverse situations that may cause trauma in childhood.

$\checkmark \quad$ It has been observed that if the mother is suffering from psychological problems, this will lead to traumatic effects on children. Therefore, providing psychological support to mothers or organizing family counseling or parent training programs may be beneficial.

$\checkmark$ Starting from the preschool period, professionals such as child development specialists, social workers, and psychologists should provide support to children by cooperating.

$\checkmark$ Within the scope of children's rights, it should be aimed to raise awareness in the society about children's right to life, right to education, and right to participate, and about their best interests. 


\section{References}

Adiguzel, V., Ozdemir, N. \&Sahin, S. K. (2019). Childhood traumas in euthymic bipolar disorder patients in Eastern Turkey and its relations with suicide risk and aggression. Nordic journal of psychiatry, 73(8), 490-496.

Beydogan Tangor, B. \& Curun, F. (2016). Psikolojik iyi olusun yordayicilari olarak bireysel farkliliklar: ozgunluk, benlik saygisi ve surekli kaygi. Egitim ve Ogretim Arastirmalari Dergisi, Cilt: 5 Sayi: 4.

Bruhn, D. C. (2010). Cyberbullying among german and dutch adolescents research on siblings' influence and factors relevant for prevention and intervention. Master's Thesis, University of Twente.

Buyukozturk, S. (2012). Sosyal bilimler icin veri analizi el kitabi. istatistik, arastirma deseni spss uygulamalari ve yorum. Ankara: Pegem Yayincilik.

Campbell, J. A., Walker, R. J. \& Egede, L. E. (2016). Associations between adverse childhood experiences, high-risk behaviors, and morbidity in adulthood. American Journal of Preventive Medicine, 50(3), 344-352.

Celebi, B. M. \&Polat, A. (2019). Çocukluk cagi travmatik yasantilarin, yetiskin baglanma stillerinin ve psikolojik iyi olusun evlilik doyumu uzerindeki etkisi. Kocaeli University Saglik Bilimleri Dergisi, 5(1), 29-34.

Christopher, J. C. (1999). Situating psychological well-being: Exploring the cultural roots of its theory and research. Journal of Counseling and Development, 77, 141- 152.

Gopalakrishnan, S. K. \&Sundram, S. (2014). Cyber bullying victimization and social anxiety among secondary school students. International Journal of Current Research And Academic Review, C. 2, S. 10, p. 55-59.

Guler, M. (2019). Universite ogrencilerinde siber zorbalik ve psikolojik iyi olusun incelenmesi. Master Thesis, Erzincan Binali Yildirim University Social Science Institude.

Isgor, I. Y. (2017). Universite ogrencilerinin psikolojik iyi olus duzeylerinin bazi degiskenler acisindan incelenmesi. Uluslararasi Turkce Edebiyat Kultur Egitim (TEKE) Dergisi, 6(1), 494-508.

Kim, H.K. \& McKenry, P.C. (2002). The relationship between marriage and psychological wellbeing a longitudinal analysis. Journal of Family. 23(8):885-911.

Martha, A. M., John, P. H., William, A. Z. \& David, P. D. (2002). Psychological distress in childhood trauma survivors who abuse drugs, The American Journal of Drug and Alcohol Abuse, 28:1, 1-13, DOI: 10.1081/ADA-120001278

Medrano, M. A., Hatch, J. P., Zule, W. A. \& Desmond, D. P. (2002). Psychological distress in childhood trauma survivors who abuse drugs. The American journal of drug and alcohol abuse, 28(1), 1-13.

Oymak, Y. C. (2017). Psikolojik iyi olus ile is doyumu arasindaki iliskinin incelenmesi. Master Thesis, Halic University Social Science Institude.

Ozen, Y. (2010). Kisisel sorumluluk baglaminda oznel ve psikolojik iyi olus (sosyal psikolojik bir degerlendirme). Dicle University Social Science Institude Journal, 4, 46-58.

Ozmete, E. (2016). Evli kadinlar ve erkekler icin psikolojik iyi olus olceginin Turkce'ye uyarlamasi. Bilig, 78, 361.

Portnova, A. A. \& Sivolap, Y. P. (2017). Substance abuse: a relationship with childhood traumas. Zhurnal nevrologii i psikhiatrii imeni SS Korsakova, 117(4), 92-95.

Reinherz, H. Z., Giaconia, R. M., Pakiz, B., Silverman, A. B., Frost, A. K. \& Lefkowitz, E. S. (1993). Psychosocial risk for major depression in late adolescence: A longitudinal community study. Journal of the American Acamedy of Child \& Adolescent Psychiatry, 32(6), 11551163. 
Roothman, B., Kirsten, D. K. \& Wissing, M. P. (2003). Gender differences in aspects of psychological well-being. South African Journal of Psychology, 33, 212-218.

Ryff, C. D. \& Singer B. (2008). Know thyself and become what you are: a eudaimonic approach to psychological well-being. Journal of Happiness Studies,(9), 13-39.

Talu, E. \& Avci, N. (2019). Annelerin ozgeci suc korkusunu etkileyen faktorlerin incelenmesi. Mehmet Akif Ersoy University Egitim Fakultesi Dergisi, (51), 262-287.

Talu, E. \& Avci, N. (2019). Ozgeci suc korkusu olceginin gelistirilmesi: gecerlik ve guvenirlik calismasi. Jass Studies-The Journal of Academic Social Science Studies, Number: 75 , Summer, $p .65-80$.

Telef, B. B. (2013). Psikolojik iyi olus olcegi: Turkceye uyarlama, gecerlik ve guvenirlik calismasi. Hacettepe University Egitim Fakultesi Dergisi, 28(28-3), 374-384.

Tuzgol Dost, M. (2004). Universite ogrencilerinin oznel iyi olus duzeyleri. Doktora tezi. Ankara: Hacettepe University. Social Science Institude.

Twaite, J. A. \& Rodriguez-Srednicki, O. (2004). Childhood sexual and physical abuse and adult vulnerability to PTSD: The mediating effects of attachment and dissociation. Journal of Child Sexual Abuse, 13(1), 17-38.

Vozmediano, L., San-Juan, C., Vergara, A. I. \& Alonso-Alberca, N. (2017). "Watch out, sweetie": The impact of gender and offence type on parents' altruistic fear of crime. Sex Roles, 77(9-10), 676-686.

Yildiz, S. (2020). Universite ogrencilerinin cocukluk cagi ruhsal travma yasantilarinin psikolojik kirilganlikla iliskisinde depresyon, anksiyete ve stresin araci rolu. Master Thesis, Marmara University Educational Science Institude.

\section{Biografical Notes:}

Bayram DELES is a Ph.D. student at Ankara University, Institute of Health Sciences, Department of Child Development. Since 2018, he has been working on child development, pre-school education and special education. The researcher has articles produced nationally and internationally.

Prof. Dr. Nilgun SARP is a lecturer at Bilgi University, Faculty of Health Sciences, Department of Child Development. At the same time, she is the dean of the faculty. Her areas of work are health education, special education, family health, and child development. The researcher has many national and international research articles and completed projects. 